\title{
News in Science
}

\section{COVID-19: A Viral Phenomenon - The Insider Report}

\author{
By: Martin Profant iD
}

\section{INTRODUCTION}

On January 9th, 2021, the Laboratory Medicine \& Pathobiology Students Union (LMPSU) hosted their annual scientific conference online, focusing on the theme: "COVID-19: A viral phenomena".

The department of Laboratory Medicine \& Pathobiology (LMP) at the Temerty Faculty of Medicine, University of Toronto is home to world-class research in the area of pathobiology, from cancer to immunopathology to neuropathology. The conference began with opening remarks from LMPSU executives Karen Mao and Ziqi Liu, followed by Dr. Rita Kandel, the chair of the department of LMP.

The topic of COVID-19 research was timely, to say the least! Invited speakers were asked to share their research and knowledge about various aspects of the COVID-19 pandemic from basic virology to treatment options, and epidemiology. The keynote speakers were Dr. Samira Mubareka and Dr. Robert Kozak; notably members of the team that was among the first to isolate the SARS-CoV-2 virus.

\section{DR. SAMIRA MUBAREKA}

Dr. Samira Mubareka is a medical microbiologist at Sunnybrook Health Sciences Centre and an assistant professor in the department of LMP at the University of Toronto. Dr. Mubareka's research focus concerns studying various aspects of respiratory viruses. Dr. Mubareka began her talk by discussing her work concerning the genome sequencing of SARS-CoV-2. Her team has sequenced over 800 genomes from patient viral samples and uploaded much of the data to GISAID, a global platform for sharing data related to COVID-19.

This form of scientific collaboration is essential explains Dr. Mubareka, because much of the genomic research on SARS-CoV-2 requires large data sets and from a diverse population in order to be able to draw meaningful conclusions. Dr. Mubareka's lab also does whole genome sequencing on the SARS-CoV-2 virus and uses their data to map evolutionary relatedness among coronaviruses as well as their origin. She also explains that this type of research is essential to cataloguing and tracking mutant strains of the virus and how they effect pathogenesis. Dr. Mubareka's lab also studied viral transmission of SARS-CoV-2 and determined that it is largely spread through environmental contact (e.g., from surfaces) rather than airborne samples. Dr. Mubareka also highlighted a project she calls "Quarantino", a study of how the virus spreads in the ICU and air ambulances.

Her team determined that invasive ventilation spreads the virus the least. These findings will help inform health experts on when and what precautions to take when handling patients with COVID-19 and how these patients infect different environments. According to Dr. Mubareka this type of research is a great example of doing science for service where questions related to practical issues are asked and investigated to yield practical.

\section{DR. ROBERT KOZAK}

Dr. Robert Kozak is clinical microbiologist at Sunnybrook Health Sciences Centre and an assistant professor in the department of LMP at the University of Toronto. In his talk, Dr. Kozak specified the pandemic research priorities: diagnostic methods, treatments, and vaccines. Firstly, in a pandemic, accurate and reliable diagnostic measures are crucial to identifying and isolate infected individuals in order to mitigate viral spread. The gold standard for the COVID-19 pandemic is the PCR test, which is highly sensitive and specific. However, PCR testing requires access to a fullyequipped microbiology lab, which poses accessibility issues for remote communities.

As such, Dr. Kozak's lab devised a portable COVID-19 test, which is now being implemented in Northern Canadian communities. Furthermore, his lab is also 
working on developing a saliva test for COVID-19, which would be an improvement compared to the current nasopharyngeal swab as the former requires a precise and unpleasant technique that, if not done properly, can easily result in false negatives. Next, Dr. Kozak and his team focused on treatments for COVID-19, notably ongoing studies concerning the efficacy of interferon- $\lambda$. Dr. Kozak's lab is also developing a COVID-19 vaccine.

Although many countries have already begun mass vaccination campaigns, Dr. Kozak stated that as a society we cannot "let our foot of the gas" - there's a chance that the current vaccines may stop being effective or the virus mutates and therefore it is important to continue development. To this end, the vaccine under development by Dr. Kozak's lab is a DNA vaccine utilizing DNA encoding for the viral spike protein of the SARS-CoV-2 to produce a memory response.

Results are promising; the DNA vaccine has shown good immunogenicity and reduces viral transmission. Dr. Kozak ended his talk by speaking to the tremendous collaboration of science in the time of COVID-19 pandemic, and how this has enabled such rapid progress. If you'd like to learn more about Dr. Kozak's work, please see our interview.

\section{DR. PRABHAT JHA}

The next speaker was Dr. Prabhat Jha, epidemiologist and professor at the Dalla Lana School of Public Health at the University of Toronto. His talk focused on epidemiological methods to study the effects of the COVID-19, both in Canada and internationally. For example, in Canada, the prevalence of COVID-19 decreases with age, whereas in India it increases. This trend can be explained by the presence of multigenerational families and crowded urban centres in India. Furthermore, Dr. Jha's work revealed that India has had much lower COVID-19 mortality than would have been expected, indicating that certain factors influencing mortality may be missing in the Indian population.

This is in contrast with Peru, where deaths have far exceeded epidemiological projections, which could be attributed to such as high rates of obesity. Overall, it was fascinating to gain insight into the field of epidemiology from one of the world's foremost epidemiologists during a pandemic.

\section{DR. KEITH PARDEE}

Dr. Keith Pardee's talk focused on synthetic biology and the response to COVID-19. Dr. Pardee is an assistant professor at the University of Toronto Leslie Dan Faculty of Pharmacy. His lab has many ongoing projects related to infectious disease diagnostics including novel sensors, deployable kits, serological assays, which are under development using cell-free methods. All his devices have the biological machinery embedded into paper which can be exposed to a sample to test for COVID-19.

This is facilitated by a toehold switch, a loop of RNA at the 5 ' end of an mRNA of a reporter gene. When the loop binds to the gene of interest (e.g., a viral gene), it falls apart and allows a ribosome to translate the reporter mRNA to produces a visual signal indicative of a positive test. Dr. Pardee's team has also integrated this technology into glucose meters to produce the ability to detect multiple infectious diseases, including COVID-19.

\section{DR. ELEANOR FISH}

Next, Dr. Eleanor Fish presented her work on interferon treatment for COVID-19. Dr. Fish is a professor of immunology at the University of Toronto and an interferon expert. Interferons are a form of immune signalling molecules that initiate an antiviral cellular state and is commonly used therapeutically. Dr. Fish explained that virtually all viruses encode for proteins that block the interferon host response. Therefore, Dr. Fish says that doctors interferon can be administered exogenously to counteract these viral anti-interferon effects and improve patient outcomes.

During the COVID-19 pandemic, she and her colleagues ran a small-scale proof of concept trial in China testing type I interferon for the treatment of COVID-19. Results revealed that interferon treatment accelerated viral clearance and recovery, as well as increased the number of CD8+ T cells (immune cells that clear virally infected cells) whilst producing little inflammation. Dr Fish's work provided compelling evidence for the use of interferon towards the treatment of COVID-19 and other infectious diseases.

Convalescent plasma therapy, a treatment for infectious diseases, is the practice of transferring blood plasma from a recovered patient to an infected or high-risk patient. This treatment is effective because plasma contains antibodies from the recovered patient which confer immunity. Two conference's speakers discussed convalescent plasma therapy.

\section{DR. MICHAEL JOYNER}

Dr. Michael Joyner, a professor at the Mayo Clinic in the United States, spoke about treating patients with convalescent plasma during the COVID-19 pandemic. He conducted a study transfusing over 94000 patients with convalescent plasma and found that this treatment reduced COVID-19 mortality. Although Dr. Joyner's 
results are encouraging, clinical trials are still required to confirm efficacy and safety.

\section{DR. JEANNIE CALLUM}

The next speaker was Dr. Jeannie Callum a professor of LMP at the University of Toronto who is involved in the coordination of the CONCOR-1 trial, which seeks to determine the efficacy of convalescent plasma therapy for COVID-19. This study involves hundreds of patients at 71 sites across 4 countries and the results will help to inform public health policy as to how to best provide convalescent plasma therapy.

\section{DR. ALED EDWARDS}

The final speaker was Dr. Aled Edwards, professor of molecular genetics at the University of Toronto and founder and CEO of the Structural Genetics Consortium. Dr. Edwards' fascinating talk began by addressing a question that has been on everyone's mind: how did we let this this COVID-19 pandemic happen? Dr. Edwards explains that scientists have known for a while that a pandemic was coming, and yet sufficient preventative measures (which would have been much more cost-effective) were not enacted. The issue, he states, is that society relies on the private sector to develop new pharmacotherapies, which is a very costly process.

Since these are profit-driven corporations, they are not keen to invest in the development of drugs that will not be used for many years to come (e.g., preventative). However, Dr. Edwards has begun working on a solution; he has founded two non-profit pharmaceutical companies, VIMI and READDI, both dedicated to developing antivirals for the next pandemic. Dr. Edwards then took us through the drug development process for an antiviral drug. He began by explaining that there are three types of drug targets for an antiviral: a conserved protein on the virus, a host protein that is needed by the virus, and the host immune response to the virus. In order for a broad spectrum antiviral to remain effective, the drug target must mutate very little, and thus mutation-prone genes must be avoided. Identified targets must then be determined to be "druggable": whether a drug molecule can access and easily disrupt the function of the viral protein.

If the foregoing two main criteria have been met, researchers can proceed to design a molecule which fits the active site of the protein in order to disrupt its function. Dr. Edwards also pressed home the point that drug discovery could be greatly improved and made cheaper (resulting in cheaper drugs for consumers) if all the scientific data was open source and drug methods were not patented. He finished his informative talk by calling to the young generation of scientists in the audience to rally behind the idea of open source scientific work and not accept the current standards of the pharmaceutical industry, as medicine is a human right and should be treated as such.

\section{CONCLUSION}

This year's LMPSU's conference COVID-19: A Viral Phenomena, as the topic was relatable to every audience member. Although we have all heard the news reports and statistics, the LMPSU conference provided students, staff, and members of the public to valuable insight into the scientific work being done to combat this pandemic and the next. Ultimately, it is scientists who will allow society to escape this dire situation and move on to better times.

The interview can be read at the following link:

https://doi.org/10.33137/juls.v15i1.37692

Martin Profant is a student at the University of Toronto and a Staff Writer for the JULS. Please address correspondence to: martin.profant@mail.utoronto.ca

\section{COMPETING INTERESTS}

No competing interests declared.

Received: February 20, 2021

Accepted: July 2, 2021

Published online: July 31, 2021 